\title{
Causality Attribution Biases Oculomotor Responses
}

\author{
Jeremy Badler, ${ }^{1,2}$ Philippe Lefèvre, ${ }^{1,2}$ and Marcus Missal ${ }^{1,2}$ \\ ${ }^{1}$ Laboratoire de Neurophysiologie, Université Catholique de Louvain, 1200 Brussels, Belgium, and ${ }^{2}$ Centre d' Ingénierie des Systèmes d'Automatique et de \\ Mécanique Appliquée; Université Catholique de Louvain, 1348 Louvain-la-Neuve, Belgium
}

When viewing one object move after being struck by another, humans perceive that the action of the first object "caused" the motion of the second, not that the two events occurred independently. Although established as a perceptual and linguistic concept, it is not yet known whether the notion of causality exists as a fundamental, preattentional "Gestalt" that can influence predictive motor processes. Therefore, eye movements of human observers were measured while viewing a display in which a launcher impacted a tool to trigger the motion of a second "reaction" target. The reaction target could move either in the direction predicted by transfer of momentum after the collision ("causal") or in a different direction ("noncausal"), with equal probability. Control trials were also performed with identical target motion, either with a $100 \mathrm{~ms}$ time delay between the collision and reactive motion, or without the interposed tool. Subjects made significantly more predictive movements (smooth pursuit and saccades) in the causal direction during standard trials, and smooth pursuit latencies were also shorter overall. These trends were reduced or absent in control trials. In addition, pursuit latencies in the noncausal direction were longer during standard trials than during control trials. The results show that causal context has a strong influence on predictive movements.

\section{Introduction}

The physicist Max Born (1949) wrote (p. 9), "Causality postulates that there are laws by which the occurrence of an entity B of a certain class depends on the occurrence of an entity A of another class, where the word 'entity' means any physical object, phenomenon, situation, or event. A is called the cause, B the effect." Born added two additional spatiotemporal constraints: first, that the cause should precede (or at least be simultaneous with) the effect. Second, that there must be some sort of spatial contact between the cause and effect, even if it is by way of a chain of intermediaries. Causality is a well established perceptual and linguistic phenomenon (Wagemans et al., 2006). However, it is not yet known whether causal context, or the expectation of a causal interaction, is capable of influencing predictive motor actions.

The first modern psychological studies of causality were performed by Albert Michotte (1946/1963). Michotte used abstract visual stimuli, such as shapes that moved and collided in various ways, and made detailed manipulations of their spatial and temporal properties. His subjects responded with verbal descriptions of the resulting "scenes," and Michotte determined whether they thought there was a causal element ("object A caused object B to move") or not. Based on numerous experiments, Michotte concluded that humans perceive causality as a Gestalt, tantamount to the way they perceive shape, motion, or other fundamental qualities in the world. Michotte's results have been replicated in con-

Received April 6, 2010; revised June 3, 2010; accepted June 24, 2010.

This work was supported by the Belgian Fonds National de la Recherche Scientifique, the Fondation pour la Recherche Scientifique Médicale, the Belgian Program on Interuniversity Attraction Poles initiated by the Belgian Federal Science Policy Office, and internal research grants from the Université catholique de Louvain (Fonds Spéciaux de Recherche, Action de Recherche Concertée).

Correspondence should be addressed to Prof. Marcus Missal, Laboratoire de Neurophysiologie, Université Catholique de Louvain, Avenue Hippocrate 54 49, 1200 Brussels, Belgium. E-mail: Marcus.Missal@uclouvain.be.

DOI:10.1523/JNEUROSCI.1733-10.2010

Copyright $\odot 2010$ the authors $\quad 0270-6474 / 10 / 3010517-09 \$ 15.00 / 0$ temporary experiments (for review, see Scholl and Tremoulet, 2000), using more quantitative measurements (Hubbard and Favretto, 2003), using infants (Saxe and Carey, 2006), and even with rhesus monkeys (Hauser and Spaulding, 2006), though the innateness of causality perception is still questioned (Hubbard, 2004; Saxe and Carey, 2006; Straube and Chatterjee, 2010).

If causality attribution is truly a built-in framework within the human mind, it should not be limited only to perception. It should also extend to the motor domain, allowing subjects to apply their putative causal analyzer to an upcoming event and generate an appropriate predictive motor response. To test this hypothesis, we measured eye movement responses to a simple stimulus (Fig. 1), where a reaction target moved after being struck by a launcher target (via an interposed tool). More predictive eye movements were generated toward the causal target, even when the movement probability of both reaction targets was identical. Increasing the movement probability of one or the other reaction target largely eliminated the direction bias toward the causal target, although movements toward the noncausal target had longer latencies. Adding a time delay between the collision with the tool and subsequent motion of the reaction target eliminated the differences in movement characteristics. A second set of experiments showed that the tool was necessary to invoke the causal bias. The results suggest that causality attribution is a Gestalt that frames predictive movements and requires cognitive effort to override.

Some results first appeared in an abstract presented at SFN 2009 (Badler et al., 2009).

\section{Materials and Methods}

For experiment 1, eight healthy subjects (three female) between the ages of 21 and 40 were used. All except subject JB were naive to the design and purpose of the study. For experiment 2, 11 healthy subjects ( 7 female) between the ages of 18 and 43 were used, all naive. All subjects gave their 


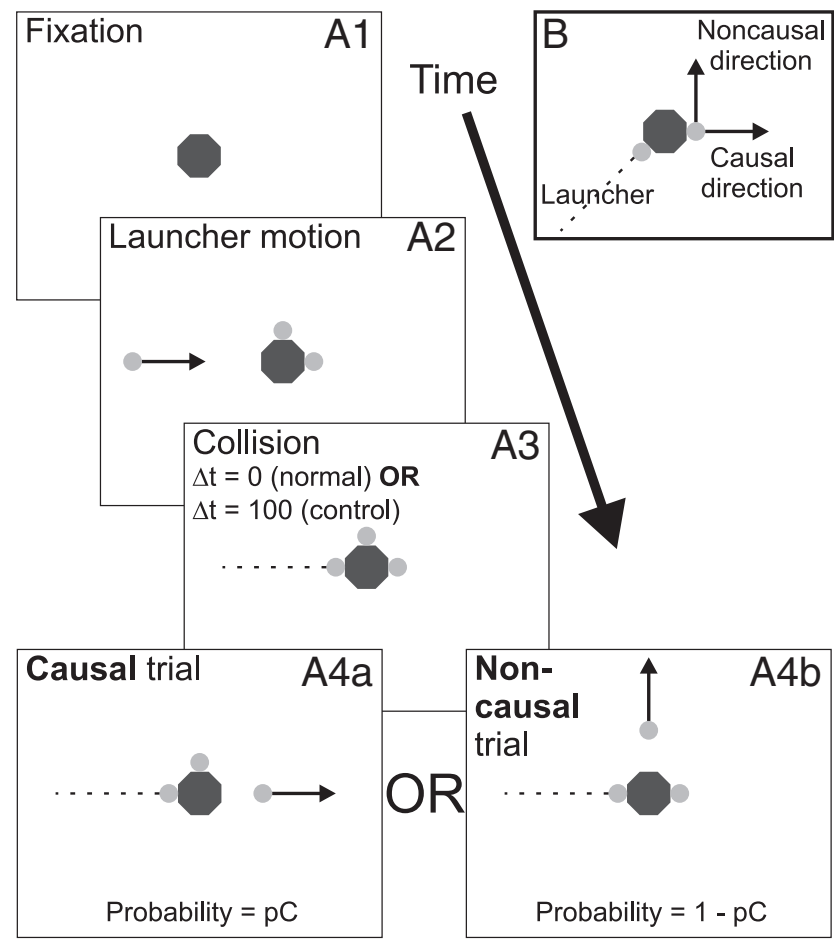

Figure 1. Schematic of the paradigm. A.1-4, Time course of experiment 1. At trial onset, the tool octagon appeared (A.1). After $500 \mathrm{~ms}$, the two stationary reaction targets appeared adjacent to the tool, as well as the launcher target which moved inward from the periphery (A.2). After an additional $370 \mathrm{~ms}$ the launcher contacted the tool (A.3). Immediately (standard trials) or $100 \mathrm{~ms}$ after the collision (delay trials), one of the reaction targets moved toward the periphery at the same speed as the launcher $(\boldsymbol{A} \cdot \mathbf{4} \boldsymbol{a}, \boldsymbol{b})$. In causal trials, the target directly opposite the launcher was the one that moved $(\boldsymbol{A} .4 \boldsymbol{a})$. In noncausal trials, the target orthogonal to the path of the launcher moved $(\boldsymbol{A} . \mathbf{4} \boldsymbol{b})$. Solid arrows indicate a current movement; dashed lines indicate a previous movement. Subjects were instructed to fixate the tool initially, then to track whichever reaction target moved after the collision. $\boldsymbol{B}$, Stimulus layout used for experiment 2. The launcher target approached at an oblique angle. After the collision, the single reaction target moved in one of the two directions indicated (50\% probability for each). There were no time-delay trials, but during no-tool blocks the octagonal tool was not visible. The fixation interval was also slightly longer $(750 \mathrm{~ms})$.

consent to participate, and all procedures were approved by the ethics committee of the Faculty of Medicine at the Universite catholique de Louvain.

Subjects were seated in a darkened room facing a dimly lit (luminance $<0.1 \mathrm{~cd} / \mathrm{m}^{2}$ ) cathode ray tube monitor (Sony Trinitron). The viewable screen area measured $41 \mathrm{~cm}$ by $31 \mathrm{~cm}$, the resolution was $800 \times 600$ pixels and the refresh rate was $100 \mathrm{~Hz}$. Their chins and foreheads rested on a padded frame $70 \mathrm{~cm}$ from the screen. Eye movements were recorded at $1000 \mathrm{~Hz}$ using an EyeLink 1000 fixed camera (SR Research Ltd.). Before each session, subjects viewed a calibration pattern consisting of a blue dot $0.37^{\circ}$ in diameter that appeared in a sequence starting at the screen center, followed by positions vertical $\pm 5^{\circ}$ and $\pm 10^{\circ}$, horizontal $\pm 5^{\circ}$ and $\pm 10^{\circ}$, and finally ending back in the center. All fixations lasted $2000 \mathrm{~ms}$. These trials were used for offline computation of offsets and gains.

Visual stimulus. The visual stimulus (Fig. 1) was similar for both experiments. It consisted of a "tool," a launcher target and one or two reaction targets. In experiment 1 (Fig. $1 A$ ), the tool was a static red octagon $1.25^{\circ}$ wide (luminance $0.2 \mathrm{~cd} / \mathrm{m}^{2}$ ) that appeared in the center of the screen at the beginning of a trial and disappeared at the end. The launcher target was a green $\operatorname{dot} 0.5^{\circ}$ in diameter (luminance $0.2 \mathrm{~cd} / \mathrm{m}^{2}$ ) that appeared after a fixation period of $500 \mathrm{~ms}$ in the screen periphery $12^{\circ}$ eccentric, and moved toward the tool in the center with a velocity of $30^{\circ} \%$ s. The two reaction targets were also green dots with the same characteristics as the launcher except that they began adjacent to the tool, and they also appeared after the fixation period. One of the reaction targets always sat on the opposite side of the tool relative to the launcher; it is referred to as the "causal target." The other reaction target sat orthogonal to the trajectory of the launcher and is referred to as the "noncausal target." After the launcher contacted the tool, one of the reaction targets moved directly away from the tool with the same velocity as the launcher. Its excursion lasted $500 \mathrm{~ms}$, which carried it off of the visible screen. The tool, launcher and inactive reaction target all remained for an additional $250 \mathrm{~ms}$. The screen was then blanked for the intertrial interval of $1500 \mathrm{~ms}$. Subjects were instructed to begin by fixating the center of the tool, then to pursue with the eyes the reaction target that moved.

For experiment 2 (Fig. $1 \mathrm{~B}$ ), there was a single reaction target, and the launcher target approached at an oblique angle. This was done to ensure that the vectorial components of the launcher motion were equal for both possible reaction target trajectories. The reaction target could move either directly away from the tool, at a $135^{\circ}$ deviation from the launcher path, or at a $135^{\circ}$ deviation to the opposite side. The former is referred to as the "causal direction," and the latter as the "noncausal direction." They are exactly analogous to the cases of experiment 1 . Subjects were instructed to fixate the reaction target (not the tool) and to follow it when it moved. The time parameters of the trials were also changed slightly (750 ms initial fixation period, $1000 \mathrm{~ms}$ intertrial interval). In addition, the color of the reaction target was changed to white to better distinguish it from the launcher, and the tool size was increased slightly $\left(1.35^{\circ}\right)$.

A single block consisted of 48 trials. The initial position of the launcher target (left, right, up, or down) was randomized. In experiment 1 , the causal target was always collinear with the launcher, but the "handedness" of the noncausal target (to the left or right of the launcher path) was also randomized in a balanced fashion for each block. The handedness was also randomized in experiment 2, by mirroring the configuration across the axis defined by the launcher target trajectory. The probability of either the causal or noncausal reaction target moving after the launcher contacted the tool was varied as an experimental parameter in experiment 1 , and this randomization was independent for all trials. The probability that the causal target moved $(\mathrm{pC})$ was one of $0.0,0.25,0.5$, 0.75 or 1.0 (subjects FP and IC were not tested with $\mathrm{pC}=0.25$ or 0.75 blocks). In experiment 2 , the probability that the reaction target moved in the causal direction (also called $\mathrm{pC}$, and analogous to the first experiment) was always 0.5 .

As a control, delay blocks were performed in experiment 1 where there was a $100 \mathrm{~ms}$ pause between when the launcher contacted the tool and the reaction target began to move. Delay blocks were performed only for $\mathrm{pC}$ values of $0.0,0.5$ or 1.0. Delays were not used in experiment 2 ; instead, in control trials the tool octagon was never shown (no-tool condition).

Experimental sessions consisted of sequential presentations of trial blocks. In experiment 1, between two and nine blocks were presented in a single session, depending on the stamina of the subject). Only one type of block was presented in a session; i.e., with a constant value for $\mathrm{pC}$ and the time delay (except for subject PD). For all subjects the first session used the standard (no-delay) $\mathrm{pC}=0.5$ block; the block type for all other sessions was selected randomly. For each condition a minimum of 5 blocks (240 trials) of data were collected.

In experiment 2, only one fixed-format session per subject was used. Subjects were first tested on five blocks ( 240 trials) of the no-tool condition, to establish a baseline response to the different geometric configurations of the moving targets. Afterward, five blocks of the standard condition were performed. As in experiment 1 , subjects were allowed to take short breaks between blocks if needed.

Eye movements. Pupil position was recorded directly using the EyeLink tracker. Subsequent analysis was performed in MATLAB (The MathWorks Inc.) using in-house software. Horizontal and vertical eye position were digitally differentiated and filtered $(25 \mathrm{~Hz}$ cutoff) to compute eye velocity. Eye acceleration was also computed by differentiation, using a $40 \mathrm{~Hz}$ cutoff filter instead. For the calibration trials, periods of stable gaze at each target position were manually selected by an operator, following which offsets and gains were computed using linear regression.

Saccade detection was based on a previous algorithm (de Brouwer et al., 2002). Briefly, saccades were marked during time intervals where the absolute value of eye acceleration exceeded $750 \% \mathrm{~s}^{2}$ for $>20 \mathrm{~ms}$. In addition, it was required that the positive and negative peak values of (signed) 
acceleration were separated by at least $30 \mathrm{~ms}$. Saccade direction was computed by averaging instantaneous movement direction over the duration of the saccade. Instantaneous movement direction $d(t)$ was defined as $d(t)=\operatorname{atan} 4\left(y^{\prime}(t), x^{\prime}(t)\right)$, where $x^{\prime}(t)$ and $y^{\prime}(t)$ are filtered horizontal and vertical eye velocity, respectively, and atan 4 is the 4-quadrant arctangent with possible values in the interval $(-180,180]^{\circ}$. Only saccades that occurred after the onset of launcher target motion were recorded.

Pursuit onset was also detected using a previous algorithm (de Hemptinne et al., 2007). Filtered velocity was required to exceed a threshold of $2 \%$ for at least $100 \mathrm{~ms}$ to be classified as pursuit. To allow for oscillations of eye velocity which sometimes occurred around the threshold, subthreshold intervals were allowed so long as they did not exceed $50 \mathrm{~ms}$ in duration. Subthreshold periods did not count toward the $100 \mathrm{~ms}$ requirement, however. Pursuit direction was calculated by averaging instantaneous direction over the first $100 \mathrm{~ms}$ of the movement.

All trials were checked by an operator to assure that saccades were detected correctly and pursuit onset could be determined unambiguously. Trials where the subject failed to follow instructions (e.g., by saccading accidentally to the launcher target), or where there was excessive noise in the eye movement trace (such as that caused by a partially closed eyelid), were rejected. The first trial of every block was also rejected outright, to preclude possible surprise effects. The total rejection rates over all subjects and conditions were $4.4 \%$ for experiment 1 and $3.4 \%$ for experiment 2.

Data analysis and statistics. For all experimental trials the $x$ - and $y$-axes were rotated or inverted as needed, such that the movement direction of the causal target was always rightward $\left(0^{\circ}\right)$ and the direction of the noncausal target was always upward $\left(90^{\circ}\right)$. All trials were aligned in time such that the motion onset time of the reaction target was zero ms. For the direction analysis (see Results), eye movements were limited to the window of $\pm 100 \mathrm{~ms}$. A single movement was selected to represent each individual trial, namely the first movement (pursuit or saccade) that occurred within the window, which served to maximize the number of data points available. Trials that did not contain any movements in the range were not included in the analysis.

For the time analysis (see Results), only smooth pursuit was used. Pursuit movements were less ambiguous than saccades, since they could only be initiated once during each trial. They were classified as being in the direction of the causal target if their direction fell within the cone of $0 \pm 30^{\circ}$, and toward the noncausal target if their direction was within $90 \pm 30^{\circ}$. Movements which did not fall in either cone were not included in the analysis.

Statistics were computed on all subjects individually, using a Bonferroni-corrected $\alpha$ of $0.0063(0.05 / 8)$ for experiment 1 and 0.0045 $(0.05 / 11)$ for experiment 2 , unless otherwise noted. For the direction analysis, movement counts were compared using a $\chi^{2}$ test $(1$ degree of freedom). For experiment 1, three types of hypotheses were tested: first, whether the counts within a session matched the $\mathrm{pC}$ value for that session ( $\mathrm{pC}=0.25,0.5,0.75)$. Second, whether the ratio of counts in a session was equal to the inverted ratio of counts for the corresponding 1-pC session ( $\mathrm{pC}=0.25 / 0.75$ and $0.0 / 1.0$ ). Third, whether the ratio of counts in a standard session was equal to the ratio of counts in the corresponding delay session ( $\mathrm{pC}=0.0,0.5$ and 1.0). Analogous tests were performed for the data of experiment 2 , though there was only one $\mathrm{pC}$ value ( 0.5$)$ along with the no-tool control condition.

For the time analysis, rank-sum tests were used to test whether the median latencies toward the causal and noncausal targets were different. For $\mathrm{pC}=0.75$ and 1.0, movement latencies in the causal direction were compared with latencies in the noncausal direction taken from sessions $\mathrm{pC}=0.25$ and 0.0 , respectively. The reverse analysis was not performed, due to the generally low numbers of movements toward an infrequent target. With the delay data $(\mathrm{pC}=0.0,0.5$ and 1.0$)$, the following three additional comparisons were performed: whether the median movement latencies in the causal direction were the same for standard and delay sessions, the analogous comparison for the noncausal direction, and a delay causal versus delay noncausal comparison analogous to the one performed for the standard sessions. Again, only tests using $\mathrm{pC}=0.5$ were needed for experiment 2 .
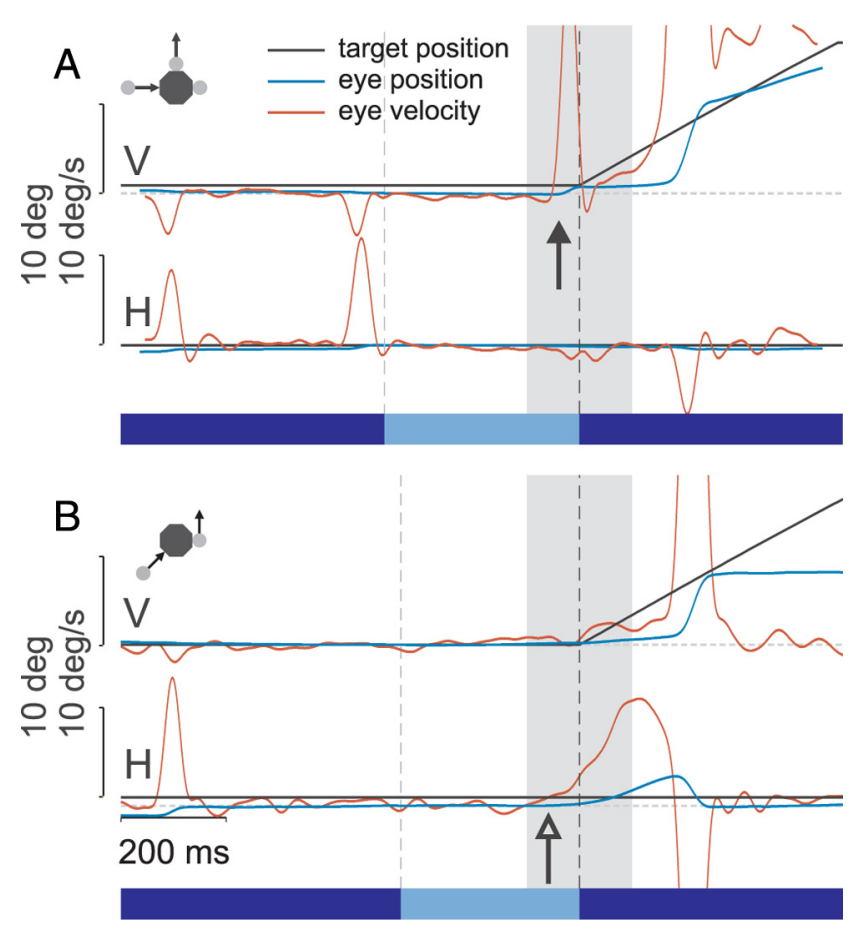

Figure 2. Typical eye movement traces during two single trials of experiments one and two. The solid bars at the bottom of each panel indicate the three phases of the trial (tool visible only, approach of the launcher target, and movement of the reaction target). Dashed vertical lines indicate the boundaries of each phase; the dashed horizontal lines indicate zero. The gray shaded region denotes the $200 \mathrm{~ms}$ window centered on the onset of reaction target motion, used for the direction analysis. For horizontal $(\mathrm{H})$ movements, right is positive; for vertical (V) movements, up is positive. The small diagrams in the upper-left corner illustrate the stimulus configuration. The tool is always centered at spatial coordinates $0,0 . \boldsymbol{A}$, Anticipatory saccadic response (filled arrow) toward the noncausal target during a noncausal trial in experiment 1. The saccade preceded the collision time, when the launcher target contacted the tool. A smooth pursuit movement was initiated shortly after contact, which included a catch-up saccade. The other saccades were small refixation movements that occurred when only the tool was visible. Subject SC. $\boldsymbol{B}$, Anticipatory pursuit (open arrow) in the causal direction during a noncausal trial in experiment 2. The subject subsequently made a corrective saccade toward the target. The launcher excursion interval is slightly shorter than in experiment 1 due to the slightly larger tool. Subject Jl.

\section{Results}

All subjects were able to follow the task instructions, although some had difficulty generating the high-velocity smooth ocular pursuit necessary to follow the targets and used predominantly saccades. The rest of the subjects used a combination of smooth pursuit and saccades. Eye movement traces from typical subjects performing the experiments are shown in Figure 2. Figure $2 \mathrm{~A}$ shows an anticipatory saccade response (filled arrow) toward the noncausal target, that began just before the launcher target collided with the tool (black dashed vertical line) in experiment 1 . Ultimately, the noncausal target was the one that moved in that trial (inset). Figure $2 B$ shows an anticipatory pursuit response in the causal direction (open arrow) for a different subject in experiment 2 . The target ultimately moved in the noncausal direction and the subject had to make a correction.

Initial direction and latency were computed for all pursuit and saccadic movements (see Materials and Methods) that began after the onset of launcher target motion. Typical movement distributions for one subject are plotted in Figure 3. It shows movements from all trials in experiment 2. The movements are plotted as latency versus direction and are sorted by type (smooth pursuit movements in $A, B$, saccades in $C, D$ ) and by condition 
(standard trials in $A, C$; no-tool trials in $B, D)$. The reference time 0 indicates the onset of the motion of the reaction target (and here, is equivalent to the collision time when the launcher strikes the tool).

The shaded gray region was defined as the "predictive zone," designed to isolate predictive movements. The lower bound $(-100 \mathrm{~ms})$ was based on the finding that in humans, for a pursuit task with a predictable $500 \mathrm{~ms}$ fixation interval, anticipatory movements usually began $\sim 100 \mathrm{~ms}$ before the onset of target motion (Heinen et al., 2005). The upper bound (+100 ms) was based on the finding that human visually guided pursuit and saccade latencies are normally $>100 \mathrm{~ms}$ (Krauzlis and Miles, 1996). The region was a good empirical fit with all of the subjects, since earlier movements were usually small refixation saccades with a range of directions, and later movements were mostly visually guided with directions tightly correlated with the causal or noncausal target. By contrast, movements within the predictive region showed intermediate dispersion, being usually in or near the quadrant defined by the two possible target choices.

There are several notable features visible in the data of Figure 3. First, predictive zone movements were dominated by smooth pursuit, especially for experiment 2 where they comprised $88.2 \%$ over all subjects and conditions. In experiment 1 , $62.3 \%$ of predictive zone movements were smooth pursuit. Broken down differently, smooth pursuit occurred during the predictive zone in $73.3 \%$ of the trials in experiment 2 . A total of $7.7 \%$ of trials had a pursuit movement that began before the predictive zone, $17.9 \%$ had pursuit that began after, and $1.1 \%$ had no observable pursuit response (only saccades). This trend is well represented in Figure $3, A$ and $B$. Predictive-zone pursuit was less predominant in experiment 1 , with only $34.6 \%$ of trials having it. A total of $4.9 \%$ of trials had prepredictive pursuit, $55.6 \%$ had postpredictive pursuit and $4.9 \%$ had no pursuit, again across all subjects and conditions.

There were also differences in the distribution of saccades between the experiments. In experiment $1,20.2 \%$ of trials had at least one saccade in the predictive zone. A total of $23.3 \%$ of trials had prepredictive saccades, $89.4 \%$ had postpredictive and $11.4 \%$ had none. In experiment 2 , only $9.6 \%$ of trials had predictivezone saccades; $7.8 \%$ had prepredictive, $97.3 \%$ had postpredictive and $4.0 \%$ had none. Note that only saccades with directions between -30 and $120^{\circ}$ were included in the tally.

\section{Direction analysis}

In Figure $3 A$ there appears to be a bias toward the causal direction, with more movements clustered around $0^{\circ}$ than around $90^{\circ}$. To quantify the bias, we examined the distribution of movement directions within the predictive zone. For each trial, a single movement was selected from inside the zone (pursuit or saccade;

\section{Standard trials}

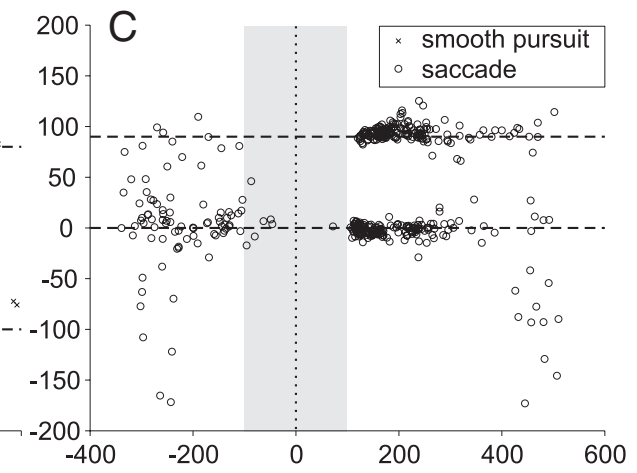

Control trials (no-tool)

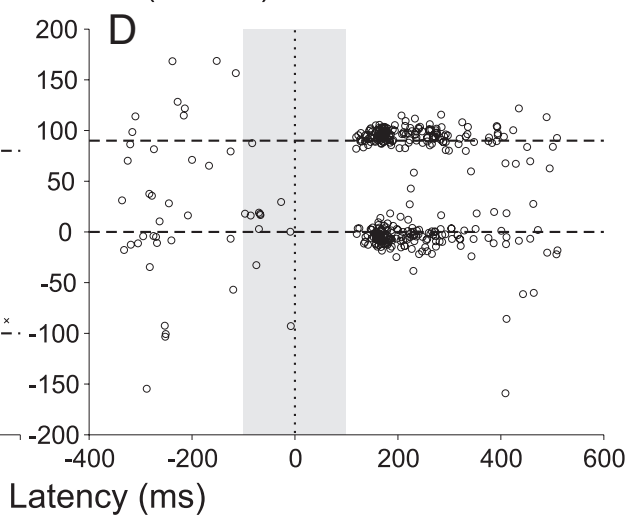

Figure 3. Movement initiation times and directions recorded from a single subject (CK) for all trials in a session of experiment 2. Dashed lines indicate the normalized direction of the causal $\left(0^{\circ}\right)$ and noncausal $\left(90^{\circ}\right)$ trajectory, and the gray shaded region

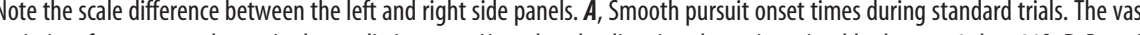
rity of movements began in the predictive zone. Note that the direction cluster is noticeably closer to 0 than $90^{\circ}$. $\boldsymbol{B}$, Pursuit 的-tool) trials. The direction bias evident in the standard trials is absent. C, Saccade onset times during to begin slightly earlier. $\boldsymbol{D}$, Saccades during no-tool trials. The early movement bias in the causal direction is no longer present, but otherwise the pattern of movements is similar to that of the standard trials.

see Materials and Methods). For experiment 1, the directions of these movements were then plotted as histogram distributions (Fig. $4 A$ ), for all causal probability values. The middle panel represents the $\mathrm{pC}=0.5$ data for one subject. Even though both reaction targets were equally likely to move, a majority of movements were directed in the causal direction. The upper panels in the figure correspond to higher probabilities of causal target motion, where predictive movements occurred almost exclusively in the causal direction. Lower panels show sessions with higher noncausal probabilities, where the movement distributions shifted to increasingly favor the noncausal targets.

The movement direction data for all subjects in experiment 1 is summarized in Figure $4 B$. Here, the fraction of initial movements toward the causal target is plotted as a function of ascending probability that the causal target would move. Individual subjects are shown as points and the group median is indicated by the solid line. If the average movement fraction reflected the actual movement probabilities, it would closely follow the dotted unity line. Instead, there is a marked deviation toward one at $\mathrm{pC}=0.5$, which represents the bias toward the causal target. Five of the eight subjects had a movement fraction significantly $>0.5$ ( $p<0.0063$; Yates' corrected $\chi^{2}$ test with Bonferroni-corrected $\left.\alpha\right)$. An additional subject was marginally significant $(p<0.05$; uncorrected $\alpha$ ). A full listing of 

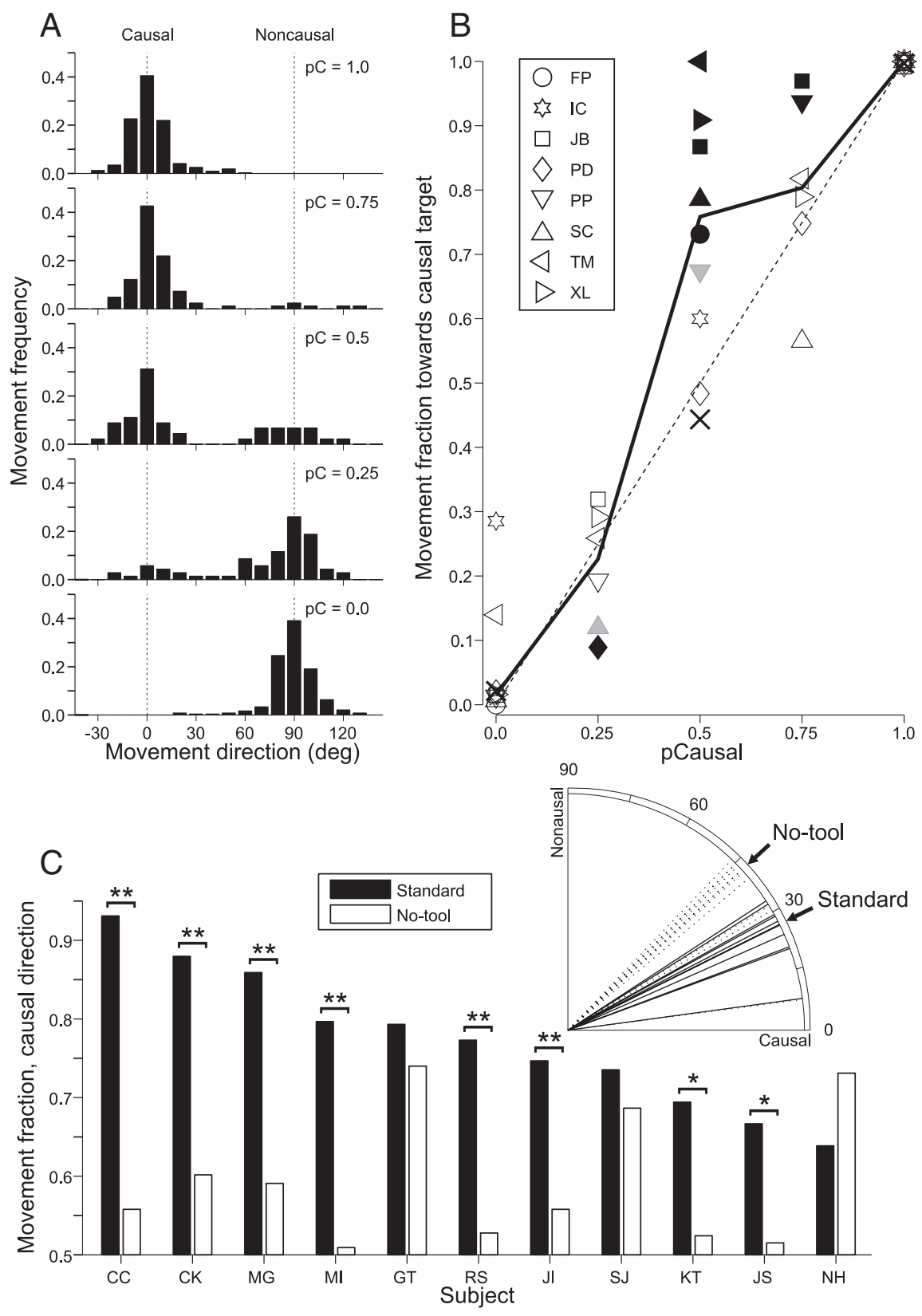

Figure 4. A, Histograms of predictive movement direction for all causal target probabilities for a single subject (SC) for experiment 1. A predictive movement was defined as the first movement (pursuit or saccade) in a trial which occurred in the interval \pm 100 ms around the onset of reaction target motion, and had a direction within $30^{\circ}$ of either the causal or noncausal target. The majority of predictive movements were directed toward the target that was more likely to move in the session. However, a disproportional number of movements occurred toward the causal target in the equiprobable case $(\mathrm{pC}=0.5$, middle panel). Dotted lines indicate the directions of the causal and noncausal targets $\left(0^{\circ}\right.$ and $90^{\circ}$, respectively). Note that for illustrative purposes, all movements within the interval $\left[-40^{\circ}, 130^{\circ}\right]$ are shown. $\boldsymbol{B}$, Fraction of initial movements directed toward the causal target for all subjects in experiment 1 , as a function of the percentage of causal trials in the session. Individual subjects are shown as symbols. The median across subjects (solid line) lies close to the equal probability line (dashed) except in the case of $\mathrm{pC}=0.5$. There is also no causal bias in the medians from the delay sessions (Xs). Filled symbols indicate significance (black, $p<0.0063$; gray, $p<0.05$ ). $C$, Fraction of initial movements in the causal direction for experiment 2. All of the fractions indicated by the black bars are significantly $>0.5(p<0.0045)$. The white bars for subjects GT, SJ and NH were also significant at this level, and the one for subject CK was marginally so $(p<0.05)$. The brackets indicate a comparison between the two conditions (standard vs no-tool control), with asterisks denoting significance $\left({ }^{* *} p<0.0045 ;{ }^{*} p<0.05\right)$. The inset shows for all subjects the distribution of median initial pursuit directions for standard and no-tool trials. Note the shift toward the causal direction in the standard condition.

movement counts and $p$-values can be found in supplemental Table 1, available at www.jneurosci.org as supplemental material.

As a control, the movement fractions for delay sessions were also examined. In delay trials there was a $100 \mathrm{~ms}$ pause between the launcher collision and reaction target motion, sufficient to greatly reduce the percept of causality (Michotte, 1946/ 1963), but the stimulus geometry was otherwise identical. Subject medians are indicated by the large Xs in Figure $4 B$. Unlike the standard sessions, the control movement fractions did not show a significant bias toward the causal target (seven of eight subjects; see supplemental Table 1, available at www.jneurosci.org as supplemental material). A direct comparison between standard and delay sessions confirmed the result: five subjects showed significant or marginally significant higher movement fractions toward the causal target during standard trials. Thus temporal proximity, one of the prerequisites of causality, is necessary for evoking the predictive direction bias.

When either the causal or noncausal target moved more than half of the time, the causal bias was substantially diminished. For $\mathrm{pC}=0.75$, only two of six subjects moved toward the causal target significantly more often than would be predicted if they were simply matching the target motion probability of the session. An analogous result was obtained for $\mathrm{pC}=0.25$, with two subjects moving more often toward the noncausal target. In addition, for each subject, the movement fraction toward the causal target at $\mathrm{pC}=0.75$ was compared with the fraction toward the noncausal target at $\mathrm{pC}=0.25$. The results were consistent with the first analysis, with four subjects showing no significant difference ( $p>0.05)$, two subjects moving relatively more frequently toward the causal target and two subjects toward the noncausal target (supplemental Table 1, available at www.jneurosci.org as supplemental material). Overall, for these probability values the subject responses were idiosyncratic, showing various degrees of influence from causal induction, block context and individual cognitive strategies.

For the extreme $\mathrm{pC}$ values $(0.0$ and $1.0)$, the initial movement directions did not show evidence of a preference for the causal target. None of the subjects showed a significant difference with the delay condition, and the cross-block comparisons were also not significant (supplemental Table 1, available at www.jneurosci.org as supplemental material; it was impossible to test the movement fraction against the block percentage directly because one of the bins would have had an expected count of zero). The certain knowledge of which target would move was apparently sufficient to suppress any inclination to follow the other, even if it was expected in the causal sense. This is not unexpected, given the 
powerful influence of cognitive expectation on eye movements (Kowler, 1989). However, the movement characteristics from the $\mathrm{pC}=0.0$ and 1.0 sessions were not identical, as a subsequent analysis will show.

Because the launcher target and causal reaction target had collinear trajectories, planned movements in the causal direction may have been facilitated simply by motion-sensitive neurons in the sensory pathway that were activated by the launcher. Experiment 2 was designed to address this. Here, the launcher target approached the tool at an oblique angle, contributing equal motion vector components in the causal and noncausal direction of the reaction target (Fig. $1 \mathrm{~B}$ ). The direction bias results for experiment 2 are plotted in Figure 4C. All 11 subjects made significantly more than half of their predictive-zone movements in the causal direction (black bars).

To rule out the possibility that subjects were simply using trajectory extrapolation to choose their initial movement direction, they were all tested on control blocks, which had the same target geometry and motion characteristics but lacked the tool. The control data are also shown in Figure $4 C$ (white bars). Although all subjects still showed some preference toward the causal direction, it was less often significant (4/11 versus 11/11). In fact, subjects usually initiated a pursuit movement in a direction intermediate to the two possible reaction target trajectories, reminiscent of the response to two simultaneously moving targets (Lisberger and Ferrera, 1997). In 8/11 cases the causal direction movement fraction was significantly higher in the standard condition than the no-tool control condition (brackets). Furthermore, the same eight subjects showed a significant difference in initial movement direction between standard and no-tool blocks (inset; rank-sum test, $p<0.0045$ ). Thus, trajectory extrapolation itself is not sufficient to explain the direction bias.

Although both pursuit and saccades were included in the direction analysis, in practice saccades had very little influence on the results. As stated earlier, experiment 2 was particularly dominated by smooth pursuit, and omitting the saccades made no qualitative difference (data not shown). Even though saccade responses were more common in experiment 1, omitting them also did not change the central result. It did cause a loss of statistical power (and thus significance) for subjects that frequently made them, such as FP. Performing the direction analysis using exclusively saccades was not possible for the majority of subjects.

\section{Timing analysis}

Reexamining Figure 3, it is apparent that not only a direction bias was present in the data. In the standard condition, movements toward the causal target appeared to occur earlier as well. To quantify this effect, we focused on smooth pursuit, which (unlike saccades) occurred only once per trial. Pursuit movements were classified as being directed toward the causal or noncausal target if they fell within a cone of $\pm 30^{\circ}$ centered on zero or 90 , respectively. Latencies of the two groups were then compared. Figure $5 A$ shows cumulative distribution curves of pursuit onset time in the standard condition, using the same dataset as Figure 3, $A$ and $B$. The median latency toward the causal target was significantly shorter, by $\sim 40 \mathrm{~ms}$ in this case (rank-sum test, $p=0.0000$ ). By contrast, the latency difference in the no-tool condition was not significantly different (Fig. 5B).

For all subjects, we compared the median latency of movements directed toward the causal target against movements directed toward the noncausal target. The results from experiment 1 are given in Figure 6. Figure $6 A$ shows a scatterplot of latencies for $\mathrm{pC}=0.5$. Five of eight subjects are significantly below the

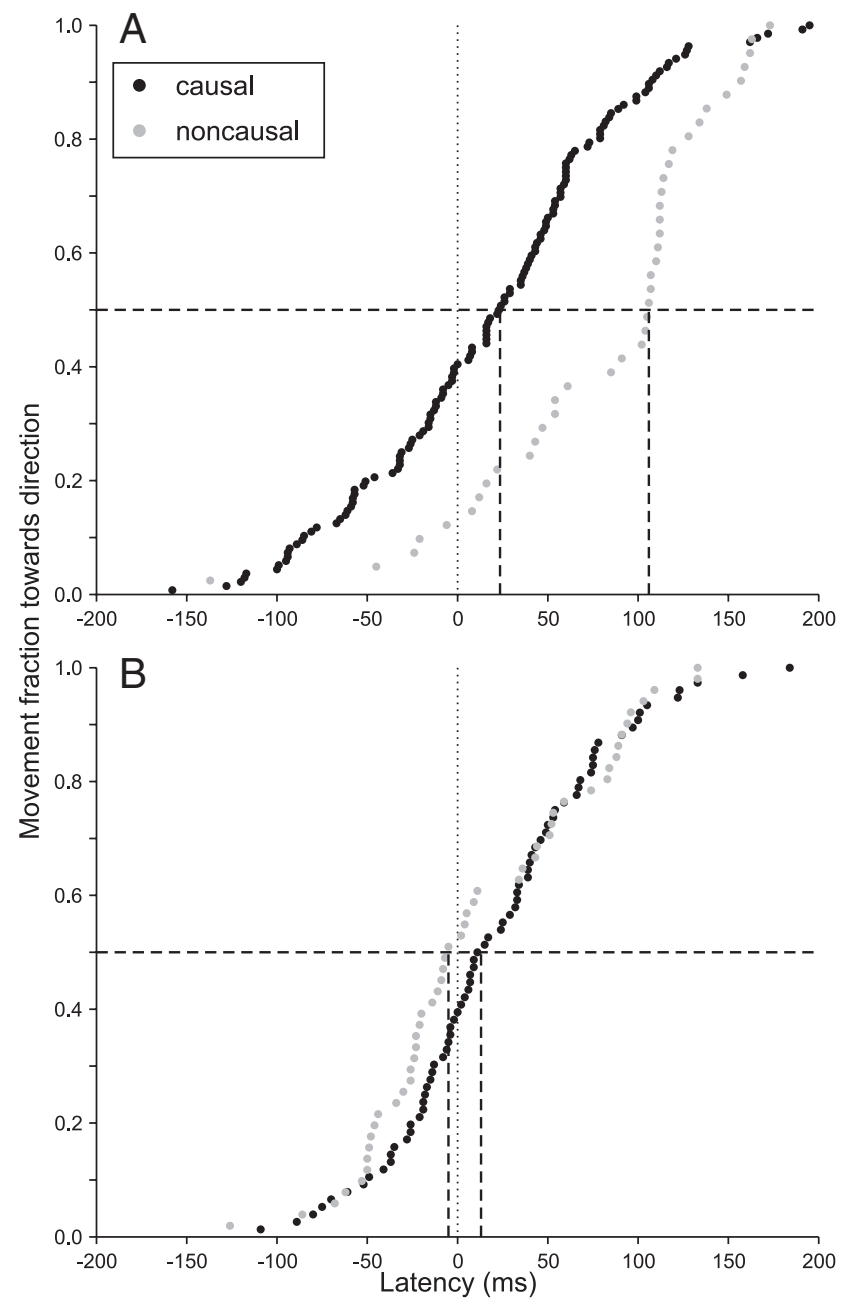

Figure 5. Cumulative frequency plots of pursuit onset time toward the causal or noncausal target, with the same data as in Figure 3 (subject CK, experiment 2). The dashed lines indicate median values. $\boldsymbol{A}$, Standard trials. Note that movements generally began earlier toward the causal target. $\boldsymbol{B}$, No-tool trials. The latency difference is no longer significant.

unity line, indicating that movements toward the causal target occurred earlier (rank-sum test, $p<0.0063$ ). On the other hand, movements toward the causal and noncausal targets were not significantly different during delay sessions for the majority of subjects (Fig. 6B). In addition, movements toward the causal target during standard sessions were significantly earlier than those movements in the corresponding delay sessions, in four subjects (Fig. 6C). Finally, there was some evidence of active inhibition in the direction of the noncausal target: half of the subjects moved significantly later toward that target in standard sessions, compared with the delay trials (Fig. 6D). The full listing of statistical comparisons can be found in supplemental Table 2, available at www.jneurosci.org as supplemental material.

To compare latencies for the remaining $\mathrm{pC}$ values, the same method was used as for the direction analysis: movements in complementary sessions were matched as necessary. For example, onset times of movements toward the causal target in a $\mathrm{pC}=$ 0.75 session were compared with onset times of movements toward the noncausal target in a $\mathrm{pNC}=0.75$ (i.e., $\mathrm{pC}=0.25$ ) session. The $\mathrm{pC} / \mathrm{pNC}=0.75$ comparisons yielded essentially a negative result, as only two subjects showed significantly earlier movements toward the causal target (supplemental Table 2, available at www.jneurosci.org as supplemental material). The 

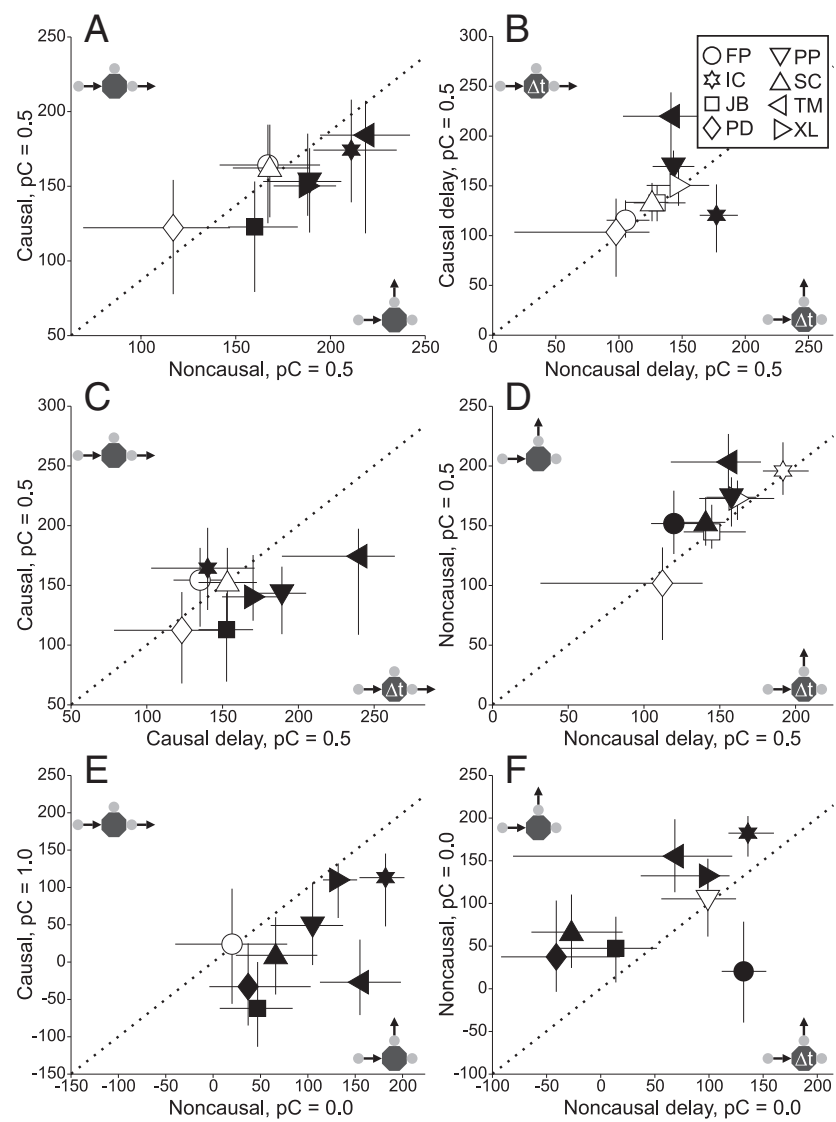

Figure 6. Difference in median movement latencies for all subjects, for $p C=0.5(\boldsymbol{A}-\boldsymbol{D})$ and $p C=0.0$ and/or $1.0(\boldsymbol{E}, \boldsymbol{F})$ for experiment 1 . Error bars show interquartile range and filled symbols indicate significance. The small diagrams next to each axis illustrate the condition represented by that axis. Note that axes are not identical. $\boldsymbol{A}$, Causal versus noncausal. The majority of subjects had shorter latencies toward causally oriented targets, shown by the points below the unity line. $\boldsymbol{B}$, Delay causal versus delay noncausal. Without the causal context there was no consistent difference between response times in the causal and noncausal directions. $\boldsymbol{C}$, Standard causal versus delay causal. Subjects had shorter latencies toward the causal target only when it appeared in a causal context; the latency reduction was eliminated by adding the time delay after collision. $\boldsymbol{D}$, Standard noncausal versus delay noncausal. Movements toward the noncausal target had a longer latency when there was a competing target that could be interpreted in a causal context. $\boldsymbol{E}$, Causal during $\mathrm{pC}=1.0$ versus noncausal during $\mathrm{pC}=0.0$. Similar results as for $p C=0.5(\boldsymbol{A})$. $\boldsymbol{F}$, Standard noncausal versus delay noncausal for $p C=0.0$. Similar results as for $\mathrm{pC}=0.5(\boldsymbol{D})$.

result is not unexpected, given the idiosyncratic nature of the same data revealed by the direction analysis. For $\mathrm{pC}=1.0$ however, the results were again strong: every subject but one had movements toward the causal target that were significantly earlier than the corresponding movements toward the noncausal target during the $\mathrm{pNC}=1.0(\mathrm{pC}=0.0)$ session (Fig. $6 E)$. Moreover, for $\mathrm{pNC}=1.0$ six subjects had significantly longer latencies toward the noncausal target in standard sessions, compared with delay sessions (Fig. $6 F$ ). The results suggest that even if direction is completely predictable, movements toward causal and noncausal targets are not identical: additional processing time is needed to counteract the effect of causal inference.

The time analysis was also performed on the data of experiment 2 , where the launcher trajectory was oblique and there was a single reaction target. For the most part, the results (Fig. 7) were in agreement with those of experiment 1 . In the primary comparison, $9 / 11$ subjects had movements in the causal direction that had significantly shorter latencies than movements in the noncausal direction (Fig. 7A). Furthermore, since the two possible
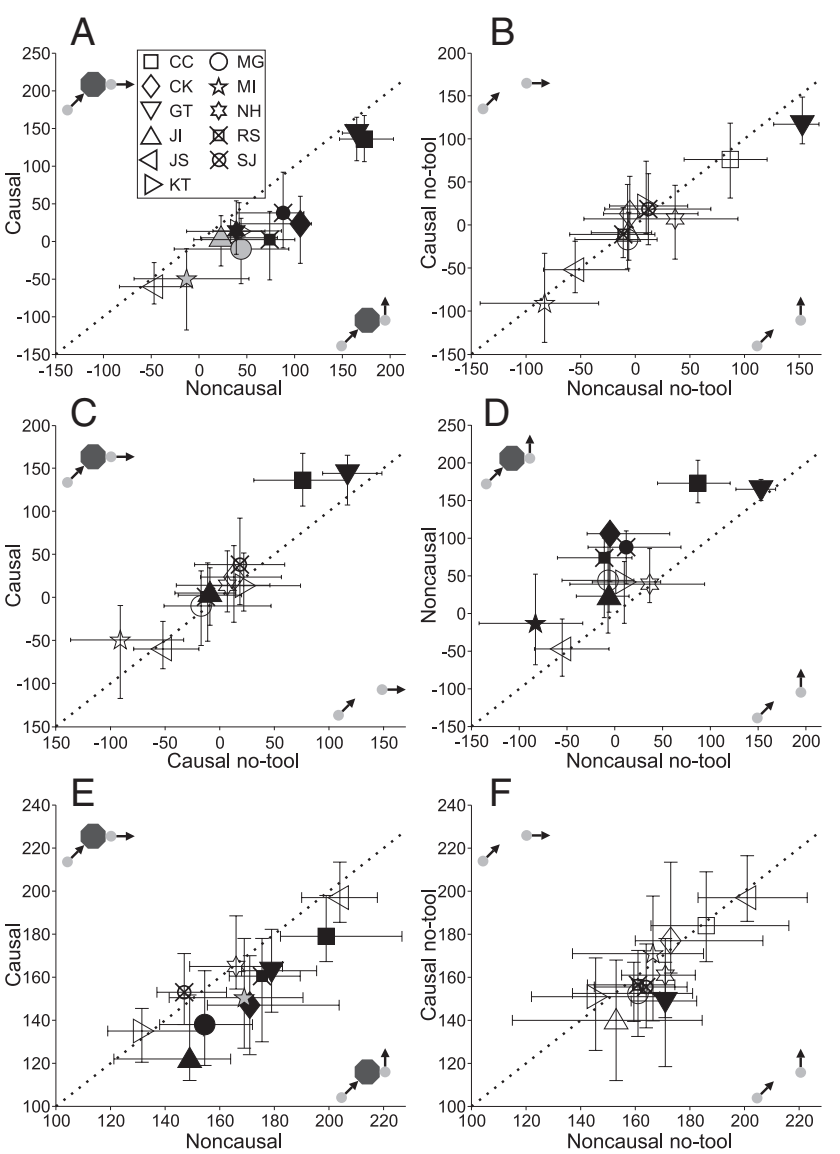

Figure 7. Difference in median movement latencies for all subjects for experiment 2. Filled symbols indicate significance (black, $p<0.0045$; gray, $p<0.01$ ); other details as in Figure 6 . Note that axes are not identical. $\boldsymbol{A}$, Causal versus noncausal. All subjects had shorter-latency movements in the causal direction, shown by the points below the unity line. $\boldsymbol{B}$, No-tool causal versus no-tool noncausal. Like the first experiment, without the causal context there was no consistent difference between response times in the causal and noncausal directions. C, Standard causal versus no-tool causal. Unlike the first experiment, causal context had little effect on movement latencies in the causal direction. $\boldsymbol{D}$, Standard noncausal versus no-tool noncausal. Also similar to what was observed in the first experiment, movements in the noncausal direction had a longer latency in the causal context, with the tool present. $\boldsymbol{E}$, Causal versus noncausal, restricted to postpredictive saccades. The latency bias for movements in the causal direction was still present. $\boldsymbol{F}$, No-tool causal versus no-tool noncausal for postpredictive saccades. As for earlier movements $(\boldsymbol{B})$, the causal bias largely disappeared in the no-tool condition.

reaction target trajectories were displaced at equal angles relative to the incoming launcher, it is unlikely that the latency difference was simply due to a perceived change in motion direction (Soechting et al., 2005). Removing the tool virtually eliminated the causal/noncausal latency difference (Fig. 7B). Unlike the first experiment, the control condition did not have much effect on movement latencies in the causal direction (Fig. 7C). Finally, increased latencies in the noncausal direction when the trial context was causal was also observed (Fig. 7D).

As with the direction analysis, variants of the timing analysis using different intervals or combinations of pursuit and saccades did not qualitatively affect the results. One such alternate is shown in Figure 7, $E$ and $F$. Here, only saccades which occurred after the end of the predictive interval in experiment 2 were tabulated. The latency result was similar: 7/11 subjects made significantly earlier saccades in the causal direction during standard trials (Fig. 7E; cf. Fig. 3C). The difference largely vanished for no-tool trials (Fig. 7F; cf. Fig. 3D). Thus the effect of causal context on latency is not limited to smooth-pursuit. 


\section{Discussion}

When a visual stimulus could be interpreted as causal, two principle effects were evident in the resulting motor responses. First, there was a bias toward making anticipatory movements in the direction of the causal target. Second, there was a tendency for movements toward the causal target to occur earlier (and conversely, for movements toward the noncausal target to occur later). When a time delay was added that eliminated the perception of causality the effects disappeared, even though the spatial configuration of the stimulus was unchanged. The causal bias did not require the launcher to be collinear with the causal target, though it did require an interposed tool between them.

Several notable results are worth considering. First, why was the causal bias not more evident for $\mathrm{pC}=0.25$ and 0.75 (Fig. $4 B$ )? For these conditions, it is important to remember that there were two contexts active simultaneously. In addition to the causal context (i.e., the expectation that the launcher collision would cause the more logical reaction target to move), there was a probability context where one reaction target was more likely to move than the other. Thus subjects effectively had a choice as to which strategy they used for choosing which target to follow first (e.g., prefer causal target, prefer target that moves more frequently, guess randomly, guess according to estimate of underlying probability distribution, etc.). Each strategy would produce its own pattern of responses, and different subjects appeared to choose different strategies. Thus the overall effect was inconsistent.

The results for experiments one and two were quite consistent, with one notable exception. In experiment 1 , movements in the causal direction during standard blocks had slightly shorter latencies than movements in the causal direction during control blocks (Fig. 6C). In fact, a general criticism of the experiment is that the collinear motion of the launcher and the causal target may have caused them to be perceived as a single motion in some observers (McKee and Welch, 1985). There is also a possibility that the launcher target activated motion-sensitive neurons in visual (Sundberg et al., 2006) or frontal cortex (Fukushima et al., 2002; Barborica and Ferrera, 2003). The subsequent decay of activation during the time delay would then explain the latency difference. In the broader sense, if the preactivation enabled movements to be programmed and executed slightly faster it would be indistinguishable from the effect of causality, which is precisely the rationale for performing experiment 2 .

By contrast, the standard versus control causal latency comparison in experiment 2 yielded no real effect. If anything, latencies in the standard condition were slightly longer than those in the control condition (Fig. 7C). This may be due to the absent tool in the control trials, which was otherwise a very salient object close to the fixation point of the subject. The lack of a fixation point is known to enhance anticipatory (Boman and Hotson, 1988) and visually guided (Krauzlis and Miles, 1996) pursuit movements.

The study of predictive eye movements in response to the viewing of a launcher display yields new insights into the way causality is perceived when two objects collide. Indeed, it provides a quantitative measure of causality attribution that does not rely on a voluntary, conscious report by the viewing subjects. In the present experiments, subjects did not have to report whether they perceive a causal relationship or not. Moreover, they had the choice to pursue one or the other target in experiment 1 . Therefore, using predictive eye movements as a tool, we measured an implicit response to the viewing of a launcher display, giving the possibility to directly assess the low-level, automatic nature of causal inference as suggested by Michotte.

The other important advantage of using predictive eye movements to study causality is that they allow the experimenter to move beyond the perceptual domain. Previous studies that did take quantitative measurements of motor actions, such as pointing location (Hubbard and Favretto, 2003), look times (Leslie, 1982; O'Connell and Dunbar, 2005) or even basic eye movements (Hindmarch, 1973), were nonetheless limited in that they considered causality perception as a post hoc phenomenon. They did not address the fundamental question of whether humans (or primates) were able to apply their inherent understanding of causality, using it to make a predictive movement before the actual causal interaction took place. Thus we can conclude that causality is not simply a descriptive phenomenon used to characterize a particular class of stimuli, but rather a fundamental part of the cognitive process with the ability to influence predictive mechanisms.

How might the expectation of a causal interaction facilitate the generation of eye movements? One possibility is via a spatial attention mechanism. Spatial attention is thought to enhance the gain of neurons tuned to a specific location, while suppressing neurons tuned to other locations (Desimone and Duncan, 1995; Verghese, 2001). In the current context, the inference of a causal relationship would enhance attention toward a putative causal target, since it is expected to move following a collision event. Concurrently, there would be a negative expectation that an alternative target might move, or that the primary target might move in a noncausal direction. The opposed expectations could result in increases or decreases in neural buildup activity, which could facilitate or retard subsequent movements as was observed (Figs. 6, 7). Support for the mechanism comes from Blakemore and colleagues (Blakemore et al., 2001), who found increased activity in visual-motion processing areas (MT/MST and others) while subjects viewed causal stimuli, as well as other studies showing that the expectation of upcoming target motion enhances premotor activity (Ferrera and Lisberger, 1997; de Hemptinne et al., 2008).

David Hume (1739/1987) hypothesized that causality inference arises from the repeated experience of correlations between events, such as when one billiard ball moves after being struck by another. On the other hand, Immanuel Kant (1781/1965) suggested that causality is an a priori category of human understanding, without which it would not be possible to make sense of the stream of events in the external world. In agreement with the latter philosophical proposal, experiments carried on by Albert Michotte and those that followed (Wagemans et al., 2006) suggested that causality perception is an innate, low-level inference imbedded in our perceptual system (Scholl and Tremoulet, 2000), just like a Gestalt. However, most previous studies of causality perception using a Michotte display used the verbal reports of subjects to assess their interpretation of what happened on the screen facing them. Verbal reports depend on high-level linguistic skills, and consequently are inevitably a subjective high-level interpretation of what happened at the perceptual level. We suggest that predictive eye movements can circumvent this important limitation to causality studies. Together, evidence suggests that humans can use the concept of causality for more than just characterizing events in the environment. Indeed, it appears to be integrated into their internal model of the world, something that facilitates visually guided actions and allows the prediction of likely event outcomes. 


\section{References}

Badler J, Lefèvre P, Missal M (2009) Causality attribution biases oculomotor responses. Soc Neurosci Abstr 35:263.27.

Barborica A, Ferrera VP (2003) Estimating invisible target speed from neuronal activity in monkey frontal eye field. Nat Neurosci 6:66-74.

Blakemore SJ, Fonlupt P, Pachot-Clouard M, Darmon C, Boyer P, Meltzoff AN, Segebarth C, Decety J (2001) How the brain perceives causality: an event-related fMRI study. Neuroreport 12:3741-3746.

Boman DK, Hotson JR (1988) Stimulus conditions that enhance anticipatory slow eye movements. Vis Res 28:1157-1165.

Born M (1949) Natural philosophy of cause and chance. Oxford: Clarendon.

de Brouwer S, Missal M, Barnes G, Lefevre P (2002) Quantitative analysis of catch-up saccades during sustained pursuit. J Neurophysiol 87:1772-1780.

de Hemptinne C, Nozaradan S, Duvivier Q, Lefèvre P, Missal M (2007) How do primates anticipate uncertain future events? J Neurosci 27:4334-4341.

de Hemptinne C, Lefèvre P, Missal M (2008) Neuronal bases of directional expectation and anticipatory pursuit. J Neurosci 28:4298-4310.

Desimone R, Duncan J (1995) Neural mechanisms of selective visual attention. Annu Rev Neurosci 18:193-222.

Ferrera VP, Lisberger SG (1997) Neuronal responses in visual areas MT and MST during smooth pursuit target selection. J Neurophysiol 78:1433-1446.

Fukushima K, Yamanobe T, Shinmei Y, Fukushima J (2002) Predictive responses of periarcuate pursuit neurons to visual target motion. Exp Brain Res 145:104-120.

Hauser M, Spaulding B (2006) Wild rhesus monkeys generate causal inferences about possible and impossible physical transformations in the absence of experience. Proc Natl Acad Sci U S A 103:7181-7185.

Heinen SJ, Badler JB, Ting W (2005) Timing and velocity randomization similarly affect anticipatory pursuit. J Vis 8:493-503.

Hindmarch I (1973) Eye movements and the perception of phenomenal causality. Psychol Belgica 13:17-23.

Hubbard TL (2004) The perception of causality: insights from Michotte's launching effect, naïve impetus theory, and representational momentum. In: Fechner Day 2004: proceedings of the twentieth annual meeting of the International Society of Psychophysics (Oliveira AM, Teixeira MP, Borges GF, Ferro MJ, eds), pp 116-121. Coimbra, Portugal: The International Society for Psychophysics.
Hubbard TL, Favretto A (2003) Naïve impetus and Michotte's "tool effect": evidence from representational momentum. Psychol Res 67:134-152.

Hume D (1739/1987) A treatise of human nature, Ed 2 (Mossner EC, ed). Oxford: Clarendon.

Kant I (1781/1965) Critique of pure reason (Smith NK, transl). London: Macmillan.

Kowler E (1989) Cognitive expectations, not habits, control anticipatory smooth oculomotor pursuit. Vision Res 29:1049-1057.

Krauzlis RJ, Miles FA (1996) Release of fixation for pursuit and saccades in humans: evidence for shared inputs acting on different neural substrates. J Neurophysiol 76:2822-2833.

Leslie AM (1982) The perception of causality in infants. Perception 11:173-186.

Lisberger SG, Ferrera VP (1997) Vector averaging for smooth pursuit eye movements initiated by two moving targets in monkeys. J Neurosci 17:7490-7502.

McKee SP, Welch L (1985) Sequential recruitment in the discrimination of velocity. J Opt Soc Am A 2:243-251.

Michotte A (1949/1963) The perception of causality (Miles TR, Miles E, transl). New York: Basic Books.

O'Connell S, Dunbar RI (2005) The perception of causality in chimpanzees (Pan spp.). Anim Cogn 8:60-66.

Saxe R, Carey S (2006) The perception of causality in infancy. Acta Psychol (Amst) 123:144-165.

Scholl BJ, Tremoulet PD (2000) Perceptual causality and animacy. Trends Cogn Sci 4:299-309.

Soechting JF, Mrotek LA, Flanders M (2005) Smooth pursuit tracking of an abrupt change in target direction: vector superposition of discrete responses. Exp Brain Res 160:245-258.

Straube B, Chatterjee A (2010) Space and time in perceptual causality. Front Hum Neurosci 4:28.

Sundberg K, Fallah M, Reynolds JH (2006) A motion-dependent distortion of retinotopy in area V4. Neuron 49:447-457.

Verghese P (2001) Visual search and attention: a signal detection theory approach. Neuron 31:523-535.

Wagemans J, van Lier R, Scholl BJ (2006) Introduction to Michotte's heritage in perception and cognition research. Acta Psychol (Amst) 23:1-19. 